\title{
Computer Integrated X-ray Systems and Intelligent Control Systems for Remote Microscopy and Power Savings.
}

\author{
R. Wuhrer ${ }^{*}$ and K. Moran ${ }^{*, * *}$
}

${ }^{*}$ Microstructural Analysis Unit, University of Technology, Sydney, Broadway, Australia.
${ }^{*}$ Moran Scientific Pty Ltd, P.O. Box 651, Goulburn, NSW, 2580 Australia.

With modern computer technologies and miniaturised circuits, SEM hardware is slowly shrinking and correspondingly reducing power consumption. Today we can walk in with almost any laptop computer and run sophisticated Electron Microscope systems with nothing more than a USB connection.

Two Jeol 733 MicroProbes, vintage 1980, were consuming a large amount of power and could not be remotely controlled. These microprobes (FIG. 1) have an excellent column design and reasonable vacuum system. Initially, a Moran Scientific fully quantitative X-Ray Mapping (XRM) hardware and software systems was installed to perform full spectrum X-ray mapping and electronically collect images. Eventually it was decided to totally upgrade the electronics by adding a full PC software control system to each microprobe.

The Moran Scientific control system operates via USB and allows control of high voltage, filament drive, gun parameters shift and tilt, spot size, astigmatism, focus, magnification, Image shift, BSE and SE brightness and contrast control, active electron beam scan control with mains synchronization (FIG. 2). The system also allows for full system integration of SEM, and multiple energy dispersive spectrometers (EDS), silicon drift detectors (SDD), Cathodoluminescent (CL) and wavelength dispersive spectrometers (WDS).

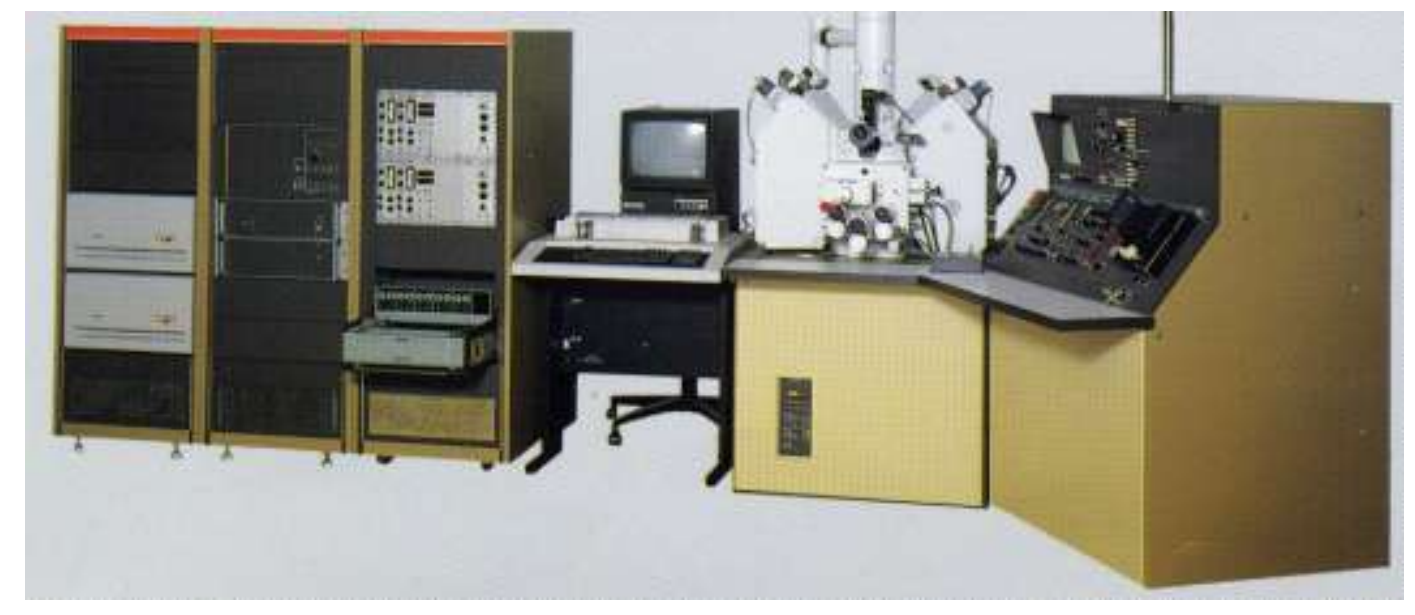

FIG. 1: Original Jeol 733 Probe that consumed 8KVA power in full operation. Image courtesy of Jeol. 

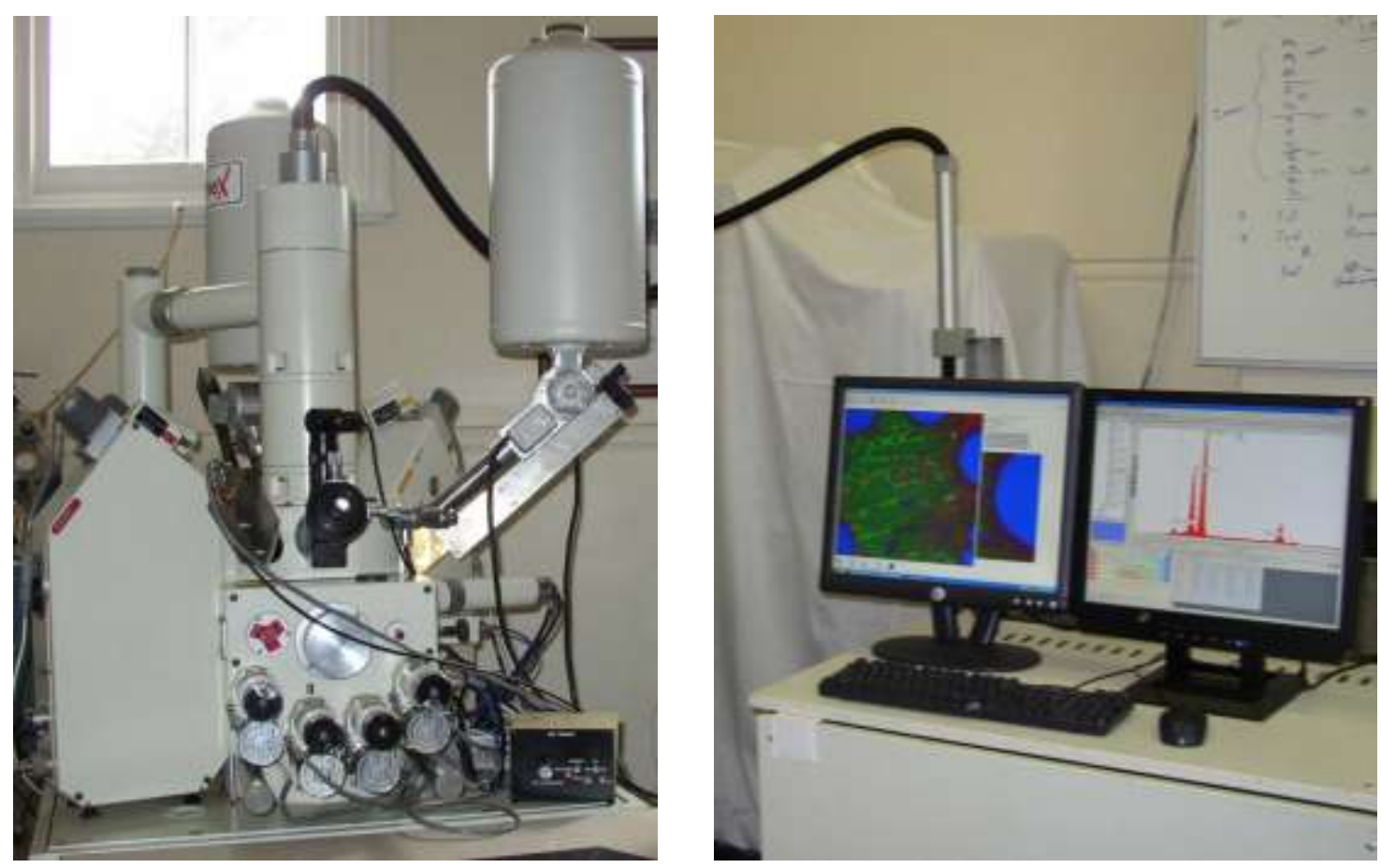

FIG. 2: Computer Integrated X-ray system. This system consumes less than 2KVA and plugs into a normal household power point.

This system allows full connection to the internet, using software such as "LogMeIn"[1]. This allows the flexibility to access and control your microprobe from anywhere. LogMeIn provides a complete remote access and management delivery solution, which also allow users to operate scientific equipment from home and easily manage and monitor all of your systems from a single web-based console.

The network speed has been found to be critical when doing this type of work, especially when running high resolution images. Our current work involves mainly monitoring overnight x-ray maps on these microprobes. Each probe is setup to maximise its ability with regard to multi EDS mapping or Multi WDS mapping, including CL and using both UTW SiLi and SDD detectors.

Remote control software has also been installed on a number of other instruments, mainly to: 1) monitor SEM conditions and performance, 2) perform overnight FEGSEM bake outs, 3) monitor EBSD maps and X-ray maps over the weekend and 4) restart new maps.

In this talk we will be discussing the changes made to the instruments and power consumption savings as well as the advantages of remote mapping using multiple EDS and WDS detectors. The talk will also discuss the use of remote control software for operating and monitoring instruments remotely.

\section{References}

